\title{
Cálculo Mental: diagnóstico de estratégias espontâneas de alunos do $6^{\circ}$ ano
}

\author{
Mental calculation: diagnosis of spontaneous strategies of \\ students of the 6th year
}

Daniel Moreira dos Santos daniel-htm@hotmail.com

\author{
Vânia Maria Pereira dos Santos-Wagner \\ profvaniasantoswagner@googlemail.com
}

\begin{abstract}
Resumo
Neste artigo apresentamos parte de uma pesquisa que tem por objetivos diagnosticar e analisar estratégias espontâneas de cálculo mental em cálculos do tipo $\mathrm{a} \pm \mathrm{b} \leq 100$. A investigação é de natureza qualitativa do tipo estudo de caso com traços da etnografia. O trabalho de campo foi realizado em uma turma de $6^{\circ}$ ano de uma escola da rede de ensino estadual do Espírito Santo. Trabalhamos com os alunos uma atividade diagnóstica composta por quatro sequências de cálculos mentais divididas em: adição e subtração com parcela e resultado menor ou igual a 5, adição e subtração com parcelas e resultado menor ou igual a 10, adição e subtração com parcelas e resultado menor ou igual a 20 e adição e subtração com parcelas e resultado menor ou igual a 100. No texto são apresentadas e analisadas as estratégias de cálculo mental de dois alunos para a sequencia de cálculos mentais com parcelas e resultado menor ou igual a 100. Constatamos que não basta o simples conhecimento de fatos numéricos. A criança deve ser levada a relacionar fatos básicos com relações numéricas mais complexas. Além disso, estratégias de cálculo mental precisam ser diretamente ensinadas, pois dificilmente surgem espontaneamente.
\end{abstract}

Palavras Chave: Diagnóstico. Estratégias espontâneas. Cálculo mental. Adição. Subtração.

\begin{abstract}
In this paper we present part of a research that aims to diagnose and analyze spontaneous strategies in mental arithmetic calculations of the type $a \pm b \leq 100$. The research is a qualitative case study type with traces of ethnography. The fieldwork was carried out in a class of 6th year of a public school of the Espírito Santo, Brazil. We work with students a diagnostic activity consists of four sequences divided into mental calculations: $a+b \leq 5$, $\mathrm{a} \pm \mathrm{b} \leq 10, \mathrm{a} \pm \mathrm{b} \leq 20$ and $\mathrm{a} \pm \mathrm{b} \leq 100$. In the text are presented and discussed strategies for mental calculation of two students to the sequence of mental calculations $a \pm b \leq 100$. We found that not just mere knowledge of numerical facts. The child should be taken to relate basic facts with more complex numerical relationships. Furthermore, mental calculation strategies need to be taught directly, as it would hardly arise spontaneously.
\end{abstract}

Keywords: Strategies . Mental calculation. Addition. Subtraction. 


\section{Introdução}

Apresentamos parte de um estudo mais amplo acerca de estratégias espontâneas de cálculo mental. Trouxemos a concepção de cálculo mental utilizada nesta pesquisa (SOWDER, 1988; PARRA, 1996; BRASIL, 1997; BUENOS AIRES, 2006) bem como as estratégias de cálculo mental identificadas por Beishuizen (1993, 2009), Beishuizen e Anghileri (1998), Lucangeli, Tressoldi, Bendotti, Bonamoni e Siegel (2003) e Thompson (2009) utilizadas aqui como categorias de análise dos dados coletados. Em seguida, descrevemos os passos do procedimento metodológico da investigação, apresentamos e analisamos os dados de dois alunos sujeitos da pesquisa utilizando por base as pesquisas acima referidas e, por fim, tecemos nossas considerações finais.

\section{Cálculo mental}

Entendemos cálculo mental como um conjunto de procedimentos que se articulam sem recorrer a um algoritmo preestabelecido para obter resultados exatos ou aproximados (SOWDER, 1988; PARRA, 1996; LINS; GIMENEZ, 1997; BRASIL, 1997; BUENOS AIRES, 2006). Os procedimentos de cálculo mental adaptam-se aos números em jogo e aos conhecimentos (ou preferências) do sujeito que as aplica. Por esta conceituação, a execução mental do algoritmo convencional não consiste em cálculo mental. Além disso, a distinção entre cálculo algorítmico e cálculo mental não reside apenas no fato de que o primeiro seja escrito e o segundo não. Como mencionamos anteriormente, o cálculo algorítmico utiliza sempre a mesma técnica para uma operação dada, qualquer que sejam os números. Em contrapartida, quando se propõe um trabalho de cálculo mental não se espera uma única maneira de proceder e estimula a criatividade.

De acordo com Parra (1996), “os procedimentos de cálculo mental se apóiam nas propriedades de numeração decimal e nas propriedades das operações, e colocam em ação diferentes relações entre os números" (p. 189). Essas estratégias utilizam as propriedades do sistema de numeração decimal (base decimal, posicional) e as propriedades aritméticas (comutatividade, associatividade, elemento neutro e elemento inverso) (SOWDER, 1988; PARRA, 1996). Albergaria e Ponte (2008), citando o trabalho de Sowder (1988), trazem a classificação dessa autora para um conjunto de características das estratégias de cálculo mental da seguinte forma: 
(i) São variáveis, o que permite que cada pessoa escolha a sua estratégia pessoal; (ii) São flexíveis, adaptando-se aos números utilizados; (iii) São holísticas, no sentido em que se lida com o número na sua globalidade, e não algarismo a algarismo; (iv) Requerem a compreensão de todo o processo de cálculo, forçando o aluno a focar a sua atenção no problema apresentado; e (v) Permitem a obtenção de resultados mais aproximados, uma vez que frequentemente se trabalha da esquerda para a direita com os números. Contudo, o cálculo mental é uma estratégia pertinente quando se trabalha com números de uma certa ordem de grandeza (ALBERGARIA; PONTE, 2008, p. 4).

\section{Estratégias de cálculo mental}

Como já nos referimos, as estratégias de cálculo mental se apóiam nas propriedades do sistema de numeração decimal e nas propriedades das operações (PARRA, 1996). São formas flexíveis de manipular as quantidades que requerem a compreensão de todo o processo (SOWDER, 1988) o que estimula o desenvolvimento do sentido numérico dos alunos (MCINTOSH; REYS; REYS, 1992; LINS; GIMENEZ, 1997; SERRAZINA, 2012). Apesar de existirem estratégias variadas de cálculo mental, existe um conjunto de estratégias que devem ser abordadas. Dentre as estratégias que envolvem adição e subtração, destacamos a decomposição de números, a compensação e o uso das propriedades das operações. Analisamos os dados coletados neste estudo à luz de estratégias desta natureza categorizadas por Thompson (2009) para as operações de adição e subtração com números até 20, categorizadas por Beishuizen (1993, 2009) e Beishuizen e Anghileri (1998) para adição e subtração com números entre 20 e 100. Além disso, utilizamos as categorias propostas por Lucangeli, Tressoldi, Bendotti, Bonamoni e Siegel (2003) para números em geral. O quadro abaixo resume as estratégias de cálculo mental categorizadas por Thompson (2009) para números menores que 20 em cálculos de adição e subtração:

\begin{tabular}{|c|c|}
\hline $\begin{array}{c}\text { Níveis de estratégias aditivas utilizadas } \\
\text { pelos alunos com números menores que } 20 \\
\text { Exemplo: } 4+5\end{array}$ & $\begin{array}{l}\text { Níveis de estratégias subtrativas utilizadas } \\
\text { pelos alunos com números menores que } 20 \\
\text { Exemplo: } 8-3\end{array}$ \\
\hline $\begin{array}{l}\text { i) contar todos: quando o aluno recorre aos } \\
\text { dedos ou material concreto para contar tudo } \\
\text { determinando o resultado de uma adição. } \\
\text { ii) contagem a partir do primeiro número: } \\
\text { o aluno conta "Quatro... cinco, seis, sete, }\end{array}$ & $\begin{array}{l}\text { i) contagem dos que sobram (count out): para } \\
\text { calcular o aluno levanta } 8 \text { dedos, abaixa } 3 \text { e } \\
\text { conta os que ficaram levantados; } \\
\text { ii) contagem para trás a partir de um número } \\
\text { (count back from): "Oito... sete, seis, cinco", } \\
\text { e para não se perder utiliza, por exemplo, os }\end{array}$ \\
\hline
\end{tabular}


oito, nove";

iii) contagem a partir do número maior: $\mathrm{o}$

aluno inicia a contagem a partir do número 5;

iv) utilização de fatos fundamentais de

adição: o aluno dá uma resposta imediata para

o cálculo;

v) cálculo com base em fatos fundamentais: o

aluno recorre a fatos fundamentais de seu

repertório de cálculo para calcular o que ainda não sabe.

vi) saltos de 10 (bridging through ten ou jumping via ten). Por exemplo, $7+6=$;

$7+3=10 ; 10+3=13$. dedos. O resultado é o último número falado.

iii) contagem para trás até (count back to):

contagem decrescente, a partir de 8 , até chegar ao 3, utilizando, por exemplo, os dedos. O resultado é a quantidade de dedos levantados.

iv) contagem até (count up): a partir do 3, o aluno conta até 8 , recorrendo, por exemplo, aos dedos;

v) utilização de fatos fundamentais de subtração e cálculo com base em fatos fundamentais,

vi) saltos de 10 (bridging through ten ou jumping via ten). Por exemplo, 12-5=; 12$2=10 ; 10-3=7$.

Quadro 1 - Estratégias de cálculo mental para números menores que 20 - adaptado de Thompson (2009)

Para cálculos de adição e subtração com números entre 20 e 100 encontramos diferentes estratégias categorizadas por Beishuizen (1993, 2009) e Beishuizen e Anghileri (1998). Essas estratégias derivam de duas ideias principais, a saber, N10 e 1010 conforme o quadro abaixo inspirado em Morais (2011) e adaptado de Beishuizen (1993, 2009) e Beishuizen e Anghileri (1998).

\begin{tabular}{|l|l|l|l|}
\hline \multicolumn{2}{|c|}{ Estratégias } & \multicolumn{1}{|c|}{ Adição: 54+38= } & \multicolumn{1}{c|}{ Subtração: 63 - 27 = } \\
\hline N10 & N10 & $54+30=84 ; 84+8=92$ & $63-20=43 ; 43-7=36$ \\
\cline { 2 - 4 } & N10C & $54+40=94 ; 94-2=92$ & $63-30=33 ; 33+3=36$ \\
\cline { 2 - 4 } & A10 & $54+6=60 ; 60+32=92$ & $63-3=60 ; 60-24=36$ \\
\hline \multirow{2}{*}{1010} & 1010 & $\begin{array}{l}50+30=80 ; 4+8=12 \\
80+12=92\end{array}$ & $\begin{array}{l}60-20=40 ; 3-7=-4 \\
40-4=36\end{array}$ \\
\cline { 2 - 4 } & $10 \mathrm{~S}$ & $50+30=80 ; 80+4=84 ; 84+8=$ & $60-20=40 ; 40+3=43 ; 43-7=36$ \\
& 92 & \\
\hline
\end{tabular}

Quadro 2 - Estratégias de cálculo mental para números maiores que 20 
Lucangeli et al (2003) observam algumas estratégias para adição e subtração tanto mental quanto escrita que se assemelham às propostas por Thompson (2009), Beishuizen (1993, 2009) e Beishuizen e Anghileri (1998). Bariccatti (2010) resume essas estratégias como COF (contagem nos dedos), CON (contagem mental a partir de um algarismo), 1010 (ou estratégia de decomposição), N10 (somente a segunda parcela é decomposta em unidades e dezenas), MA (algoritmo mental da direita para a esquerda), C10 (formação de dez unidades, por exemplo, 43+6=(43+7) -1;43-7=(43-3)-4, AUTO (cálculos automáticos ou recuperação de resultados).

\section{Procedimento metodológico}

O presente texto traz parte de uma pesquisa de mestrado de natureza qualitativa pautada na metodologia de estudo de caso do tipo etnográfico (FIORENTINI; LORENZATO, 2006; ANDRÉ, 2008). O recorte traz um episódio de aula do dia 13 de agosto de 2013 e entrevistas com dois alunos da turma pesquisada. As entrevistas aconteceram nos dias 02 e 05 de setembro de 2013. Na aula do dia 13 de agosto de 2013 conduzimos a aplicação de uma sequência de cálculos mentais em uma turma de $6^{\circ}$ ano de uma escola da rede estadual de ensino do estado do Espírito Santo. A turma possui 15 alunos com faixa etária entre 11 e 14 anos. Do início das observações até a data de aplicação da atividade foram trabalhadas as quatro operações com atividades de resolução de problemas e do tipo "arme e efetue" com algoritmos convencionais. Neste estudo, tínhamos os objetivos de diagnosticar repertório de cálculo do tipo $\mathrm{a} \pm \mathrm{b}=5, \mathrm{a} \pm \mathrm{b} \leq 10, \mathrm{a} \pm \mathrm{b} \leq 20$ e $\mathrm{a} \pm \mathrm{b} \leq 100$ e analisar estratégias dos alunos na resolução dos cálculos mentais que surgiram espontaneamente. Neste texto queremos responder ao questionamento: Quais estratégias de cálculo mental os alunos utilizam em cálculos de adição e subtração do tipo $a \pm b \leq 100$ ? A análise dos dados nos possibilitou ver alguma relação entre os números, a operação aritmética e a estratégia escolhida (Quadro 3).

\section{A atividade de pesquisa}

Organizamos a atividade em quatro sequências. Cada sequência constitui-se de vários grupos com cálculos semelhantes. Trazemos neste texto apenas parte da sequência 4 aplicada no dia 13 de agosto de 2013. Cada aluno recebeu uma folha de respostas onde deveria apenas registrar a resposta de cada cálculo. O cálculo deveria ser realizado mentalmente sem auxílio 
de nenhum registro de cálculos parciais. Ao final de cada folha de respostas existiam dois campos de respostas para serem preenchidos pelos alunos: "o que eu percebi de parecido ou diferente nos cálculos foi" e "para calcular mentalmente eu”. Com essas respostas queríamos obter dados das reflexões de cada aluno sobre a própria maneira de calcular mentalmente. Trazemos alguns grupos de cálculo trabalhados na sequência 4, a saber, na tarefa 4.e.2 (dezenas e unidades com unidades - adição com reserva e subtração com empréstimo: 45+7; $53+8 ; 39+7 ; 24+9 ; 45-7 ; 83-8 ; 67-9 ; 94-8$ ), tarefa 4.f.1 (dezenas e unidades com dezenas e unidades - dobros: $25+25 ; 27+27 ; 33+33 ; 39+39 ; 46+46$ ) e tarefa $4 . f .2$ (dezenas e unidades com dezenas e unidades - operações em geral: $34+55 ; 23+76 ; 67+23 ; 12+78 ; 14+47 ; 67-26$; $74-18 ; 97-35 ; 89-55 ; 88-39)$. Fornecemos de 20 a 30 segundos para que os alunos fizessem o registro de cada resposta. Durante o tempo dado repetíamos o cálculo pelo menos três vezes. Em vários momentos foi necessária a repetição do cálculo para os alunos após o tempo determinado. A maioria das vezes por não terem escutado corretamente, outras vezes por terem ficado para trás em algum cálculo.

\section{Apresentação e análise de dados}

Nesta seção trazemos os procedimentos e respostas dos alunos que participaram das três aulas de cálculo mental referentes à atividade diagnóstica. Ao todo oito alunos participaram das três aulas de aplicação da atividade. Abaixo trazemos os procedimentos de Luizza e Douglas. Estes nomes são fictícios e escolhidos pelos próprios alunos da turma.

\section{A aluna Luizza}

De modo geral Luizza não teve um bom desempenho nas tarefas de cálculo mental embora tenha acertado quase todas as respostas da atividade. Dos 132 cálculos realizados obteve apenas 11 erros. Na sequência 4, Luizza afirma não ter conseguido calcular mentalmente, pois nunca tinha feito esse tipo de atividade. A observação da aluna está de acordo com o que dizem os estudos de Beishuizen e Anghileri (1998). Esses autores afirmam que a falta de uma prática regular com atividades de cálculo mental que favoreçam o aprendizado e a memorização de fatos fundamentais faz com que vários alunos continuem recorrendo a estratégias de contagem, como vemos nos dados de Luizza. A aluna não possui procedimentos eficientes de cálculo mental porque nunca efetuou rotineiramente atividades desta natureza. 
Disse que no começo estava fácil (se referia aos cálculos da tarefa 4.a aos cálculos da tarefa 4.e.1), mas nos cálculos finais (a partir dos cálculos propostos na tarefa 4.e.2) a aluna alega que não dava nem para utilizar os dedos como apoio. As estratégias de contagem, com o apoio dos dedos (do tipo COF) ou sem o apoio dos dedos (do tipo CON), mostraram-se ineficientes para os cálculos com números da ordem de grandeza da sequência 4 (números maiores que 20 e menores que 100). A tentativa de usar estratégias de contagem a partir da tarefa 4.e.2 revelou que Luizza se apoiou quase exclusivamente na estratégia de cálculo exposta por Thompson (2009) de “contagem a partir do número maior”. Luizza não mobilizou estratégias de "utilização de fatos numéricos de adição" e também não usou estratégias de "cálculo com base em fatos numéricos", o que seria mais adequado a partir desse grupo de cálculos. Isso se deve a não familiaridade de Luizza com as tarefas matemáticas da sequência 4.

Luizza não mobilizou o conhecimento de fatos fundamentais (estratégia AUTO) para a realização de cálculos mais difíceis como os cálculos da sequência de tarefas 4.f.2 (LUCANGELI et al, 2003; THOMPSON, 2009). Isso ficou evidenciado em seus registros quando afirmou ter utilizado os dedos (COF) (LUCANGELI et al, 2003). Dos onze cálculos errados seis eram de subtração e cinco de adição. Dos cálculos de adição três eram cálculos de dobros onde respondeu: $39+39=48$ (a aluna adicionou as unidades resultando em 18 e em seguida adicionou uma dezena a três dezenas totalizando quatro dezenas, esquecendo-se de adicionar as outras três dezenas - uma tentativa de utilização da estratégia de algoritmo mental MA), $46+46=62$ (é possível que a aluna tenha pensado da seguinte forma: somou as unidades totalizando 12, adicionou uma dezena com três dezenas mais três dezenas totalizando 9 dezenas, mas por falta de atenção registrou o algarismo invertido, isto é, o algarismo 6 - também tentou usar a estratégia MA neste cálculo) e $19+19=32$ (calculou $9+$ 9 incorretamente como 12 em uma tentativa de usar a estratégia MA) (LUCANGELI et al, 2003). Os outros dois cálculos de adição eram da sequência 4.f.2 (dezenas e unidades com dezenas e unidades): $34+55=88$ e $14+47=60$. Erros como esses dois últimos são comuns quando os alunos utilizam os dedos no auxílio à contagem um a um, pois começam a contar a partir da parcela e não de seu sucessor. Por exemplo, ao efetuar $5+4$ alguns alunos contam “5, 6, 7 e 8”, ao invés de “6, 7, 8 e 9". Além disso, muitas vezes utilizam a combinação de duas estratégias: algoritmo mental e contagem nos dedos (MA e COF) ou algoritmo mental e contagem mental (MA e CON) o que atrapalha ainda mais a resolução do cálculo. A maioria desses cálculos de adição possuía reserva o que dificulta o uso da estratégia MA 
(LUCANGELI et al, 2003) levando o aluno ao erro e à perda de sentido numérico (BEISHUIZEN, 1993). Dos cálculos de subtração dois eram da sequência de tarefas 4.e.2 (De dezenas e unidades com unidades - subtração com empréstimo): $45-7=34$ e $67-9=53$. Os demais cálculos subtrativos pertenciam à sequência de tarefas 4.f.2: $67-26=42,74-18=$ $54,89-55=40$ e $88-39=56$.

No dia 22 de agosto de 2013 demos o retorno à Luizza de seus acertos e fizemos questionamentos sobre seus procedimentos de cálculo mental em um cálculo de cada grupo onde havia errado. Perguntamos a ela como fez, ou faria para calcular 45 - 7 (cálculo proposto em 4.e.2). Luizza disse que "chuta" um valor, adiciona 7 e verifica se o total é igual a 45. Perguntamos a ela porque experimentou 34 e não o número 20, por exemplo. Luizza disse: "porque o 20 não iria passar nem perto!", o que mostra que sua escolha não é totalmente arbitrária. Observamos através da resposta de Luizza que a aluna tem noção de estimativa e julga a razoabilidade de um número enquanto possível resultado (MCINTOSH; REYS; REYS, 1992; LINS; GIMENEZ, 1997; SERRAZINA, 2012). Fez rapidamente uma estimativa de que $20+7$ é menor que 38. Contudo, a aluna não tem uma estratégia eficiente para calcular com exatidão $45-7$, como por exemplo, primeiro retirar todas as unidades da primeira parcela: $45-5=40$ e em seguida efetuar $40-2=38$, que seria uma estratégia de saltos em 10 (THOMPSON, 1999; TREFFERS, 2008) ou do tipo C10 (LUCANGELI et al, 2003). Luizza fez novamente o cálculo e verificou que a resposta deveria ser maior do que 34 , já que $34+7=41$. Disse em seguida 38 , verificando que ao acrescentar 4 ao total $(41+4=$ 45) poderia acrescentar 4 à primeira parcela e obter o resultado $(34+4=38)$, mostrando o uso de uma estratégia do tipo compensação que se apóia no desenvolvimento de um cálculo intermediário. Não encontramos esta estratégia categorizada na literatura que tivemos acesso.

Perguntamos a ela como faria para calcular $39+39$ e Luizza nos respondeu que faz o algoritmo convencional na mente (MA) (LUCANGELI et al, 2003). Daí resultaria que "9 + 9 $=18,1+3=4$, então é 48 ". Luizza se esqueceu da dezena do outro número não se dando conta novamente que deveria adicionar uma dezena com 3 dezenas mais 3 dezenas. Quando perguntamos a ela como faria para calcular $34+55$ disse que faria do mesmo jeito, isto é, executando o algoritmo na cabeça (MA). Ao fazer o cálculo novamente, Luizza disse que a resposta deveria ser 89 e não 88 como ela havia registrado na atividade diagnóstico. Luizza também utilizou os dedos para contar um a um 4 unidades mais 5 unidades e 3 dezenas mais 5 dezenas realizando a contagem a partir do primeiro número conforme o nível ii para adição 
identificado por Thompson (2009) e conforme estratégia COF de Lucangeli et al (2003). Vemos que a aluna usa uma combinação das estratégias de algoritmo mental e contagem nos dedos. O uso dessa combinação de estratégias não foi categorizado pelas pesquisas que tivemos acesso. Luizza mexia timidamente um dedo de cada vez da mão esquerda sobre o antebraço direito. Perguntamos a Luizza se achou que ditamos muito rapidamente os cálculos e ela disse que só um pouco rápido. Não identificamos nos procedimentos de Luizza estratégias de cálculo mental complexas do tipo N10 e 1010 para adição e subtração conforme categorização proposta por Beishuizen (1993, 2009), Beishuizen e Anghileri (1998) e Lucangeli et al (2003) com parcelas entre 20 e 100.

\section{O aluno Douglas}

O aluno Douglas acertou 121 cálculos de 132. Seus onze erros se concentraram na sequência 4, principalmente no grupo de cálculos 4.f.2. Na tarefa de cálculos 4.e.2 (De dezenas e unidades com unidades - adição com reserva e subtração com empréstimo) Douglas registrou $53+8=71,94-8=82$; na tarefa 4.f.2 (De dezenas e unidades com dezenas e unidades Operações em geral) $12+78=61,14+47=40$ (nestes dois cálculos de adição colocamos a primeira parcela menor do que a segunda parcela. Vemos que as duas respostas do aluno trazem resultados menores que a segunda parcela. Acreditamos que Douglas levou em conta que as respostas deveriam ser maiores que a primeira parcela, mas não atentou para o fato de que também deveriam ser maiores que a segunda parcela), $67-26=35,74-18=62,97-35$ $=34,89-55=97,88-39=51$. Douglas não consegue ou não sabe explicar como pensou e como efetuou estes cálculos mentais. Douglas afirma que tem as contas armadas na cabeça, isto é, executa o algoritmo convencional mentalmente conforme estratégia MA (LUCANGELI et al, 2003). Como afirma Beishuizen (2001) esse tipo de estratégia mental acarreta perda no sentido de número durante o cálculo. Por exemplo, $89-55=97$, o aluno não percebe que o resultado foi maior que o minuendo. Douglas afirma ainda que quando está com dúvidas conta nos dedos (COF) (LUCANGELI et al, 2003; THOMPSON, 2009).

No dia 02 de setembro de 2013 entrevistamos Douglas para entendermos seus procedimentos de cálculo. Na tarefa de cálculo 4.e. 2 perguntamos como resolveria $53+8$. Douglas disse que faria $8+3=11$ e $50+11=61$ de modo semelhante à estratégia de cálculo 1010 proposta no modelo de Beishuizen (1993, 2009), Beishuizen e Anghileri (1998) e Lucangeli et al (2003). Perguntamos a ele como faria 94 - 8. Douglas disse que nas subtrações utilizava os dedos. 
Estendeu os dez dedos sobre a mesa e dobrou um por um, recitando números em ordem decrescente (estratégia count back from) (THOMPSON, 2009) e COF (LUCANGELI et al, 2003), até o oitavo dedo quando encontrou o número 86. Disse que deve ter errado porque estava nervoso. Embora esta não seja uma estratégia de cálculo que demonstre maturidade numericamente, Douglas a executou com segurança. Sua justificativa para os erros parece razoável uma vez que um desequilíbrio emocional (ansiedade, nervosismo) atrapalha o desempenho dos alunos em atividades avaliativas e não rotineiras como a atividade de pesquisa (GOMÉZ CHACÓN, 2003). Douglas mobilizou estratégias complexas de cálculo mental para adição ao passo que nas subtrações com empréstimo recorreu a estratégias de contagem com apoio dos dedos (COF) (THOMPSON, 2009; LUCANGELI et al, 2003). Conforme Buys (2008) o uso de estratégias mais avançadas não exclui do repertório de cálculo o uso de estratégias mais primitivas.

Nas tarefas de cálculo 4.f.2 perguntamos a Douglas como faria $12+78$ que nos disse que calcularia $10+70=80$ e $8+2=10$. Por fim, faria $80+10=90$ conforme estratégia 1010 (BEISHUIZEN, 1993, 2009; BEISHUIZEN; ANGHILERI, 1998; LUCANGELI et al, 2003). Apontamos para o resultado 61 que havia registrado e perguntamos o que ele achava deste número em relação à segunda parcela (78). Ele disse que o número 61 é muito menor do que 78 e não poderia ser a resposta. Questionamos o que ele imaginava que o teria levado ao erro. Douglas disse: “Acho que fiz de menos. Não?”. Perguntamos: “Se fosse de menos (subtração) quanto daria?”. Douglas disse $78-12 \ldots 70-10=60$ e $60-8=52,52-2=50$, utilizando uma estratégia de decomposição semelhante a 1010. Pedimos para que fizesse novamente. Queríamos nos certificar de seu procedimento. Douglas repetiu o mesmo processo.

Após nossa intervenção pedimos a ele que calculasse $67-26$, porque havia registrado o número 35 na folha de respostas. Douglas fez $60-20=40$ e disse agora vou fazer no dedo 7 $-6=1.40+1=41$. O que mostra que Douglas entendeu e utilizou a estratégia 1010 adequadamente mesmo que com auxílio dos dedos (COF) em $7-6=1$ (BEISHUIZEN, 1993, 2009; BEISHUIZEN; ANGHILERI, 1998; THOMPSON, 2009; LUCANGELI et al, 2003). Durante o diálogo Douglas mostrou-se bastante seguro resolvendo os cálculos com estratégias eficientes. Questionamo-lo por que achava que tinha errado estes cálculos que mostramos. Douglas disse que ficou nervoso durante a atividade e acabou se perdendo nos cálculos. Gómez Chacón (2003) ressalta que "as reações emocionais são o resultado de discrepâncias entre o que o sujeito espera e o que ele experimenta no momento em que a reação se produz" 
(p. 86). A atividade de cálculo mental na escola foi novidade para Douglas assim como para Luizza. Por isso, a dificuldade experimentada nas tarefas de cálculo desencadeou reações emocionais de medo, tensão e ansiedade nesses alunos.

\section{Considerações finais}

É interessante investigar as estratégias espontâneas de cálculo mental, porque se evidencia a forma como o indivíduo procede autonomamente. Ao invés de simplesmente darmos um algoritmo ou um caminho de solução, analisamos a estratégia que a criança lançou mão e inferimos questões sobre a sua aprendizagem acerca de números e operações. No quadro abaixo resumimos as estratégias principais mobilizadas por Luizza e Douglas nas três tarefas de cálculo analisadas separadas pela operação de adição e subtração.

\begin{tabular}{|l|l|l|l|}
\hline & $\begin{array}{l}\text { Dezenas e unidades com } \\
\text { unidades - adição com } \\
\text { reserva e subtração com } \\
\text { empréstimo }\end{array}$ & $\begin{array}{l}\text { Dezenas e unidades } \\
\text { com dezenas e unidades } \\
\text { - dobros }\end{array}$ & $\begin{array}{l}\text { Dezenas e unidades com } \\
\text { dezenas e unidades - } \\
\text { operações em geral }\end{array}$ \\
\hline $\begin{array}{l}\text { Luizza } \\
\text { dedos (COFão: contagem nos } \\
\text { parcela maior. } \\
\text { Subtração: “chuta" um da } \\
\text { valor para a resposta. }\end{array}$ & $\begin{array}{l}\text { Adição: Algoritmo } \\
\text { mental (MA) e } \\
\text { Contagem nos dedos } \\
\text { (COF) a partir da parcela } \\
\text { maior. }\end{array}$ & $\begin{array}{l}\text { Adição: Algoritmo mental } \\
\text { (MA) e Contagem nos } \\
\text { dedos (COF) a partir da } \\
\text { parcela maior. } \\
\text { Subtração: Algoritmo } \\
\text { mental (MA) e Contagem } \\
\text { nos dedos (COF) } \\
\text { (contagem até - count up). }\end{array}$ \\
\hline $\begin{array}{l}\text { Douglas } \\
\text { Subtração: contagens nos } \\
\text { (COF). }\end{array}$ & $\begin{array}{l}\text { Adição: Decomposição } \\
\text { das duas parcelas (1010). }\end{array}$ & $\begin{array}{l}\text { Adição: Decomposição } \\
\text { das duas parcelas (1010). }\end{array}$ & $\begin{array}{l}\text { Adição: Decomposição das } \\
\text { duas parcelas (1010). } \\
\text { Subtração: Decomposição } \\
\text { das duas parcelas (1010); } \\
\text { contagens nos dedos } \\
\text { (count back from) (COF). }\end{array}$ \\
\hline
\end{tabular}

Quadro 3 - Resumo das estratégias de Luizza e Douglas 
A análise dos dados nos permitiu ver que o nível de eficiência da estratégia de cálculo mental utilizada aumenta de acordo com o domínio de fatos numéricos fundamentais. Esse domínio de fatos básicos favorece a execução de cálculos mais complexos. Porém, não basta o simples conhecimento de fatos numéricos. A criança deve ser levada a relacionar fatos básicos com relações numéricas mais complexas por meio de atividades de cálculo mental desenvolvidas rotineiramente. Desta forma, os alunos obterão familiaridade com as estratégias. Vemos que Luizza conhecia alguns fatos, no entanto, não estabeleceu as relações numéricas necessárias para utilizar esse conhecimento como base para cálculos mais avançados. Isso fez com que a aluna não abandonasse estratégias de contagem nos dedos e o uso do algoritmo mental. Douglas utilizou durante a entrevista preferencialmente a estratégia de decomposição (1010), mas nas subtrações revelou insegurança realizando contagens nos dedos. Isso nos mostrou que possivelmente a subtração não foi trabalhada de maneira suficiente e eficiente com este aluno que, já dominou pelo menos um procedimento mental avançado para a operação de adição. É possível que Douglas não possua a operação de subtração completamente ativa, uma vez que uma das condições para que isto ocorra seja o conhecimento de fatos numéricos básicos da operação aritmética mencionada. Além do mais, durante a atividade de pesquisa, devido ao nervosismo, Douglas fez uso do algoritmo mental (MA) várias vezes. Constatamos que a insegurança e a ansiedade diante de uma tarefa não rotineira fazem com que alguns alunos recorram a estratégias que possuam maior segurança, como os procedimentos ensinados pela escola (algoritmos) e as contagens, mesmo que estes procedimentos sejam mais trabalhosos (GOMÉZ CHACÓN, 2003).

Através da análise dos dados chegamos a alguns resultados que ainda não identificamos na literatura sobre cálculo mental. Vemos que Luizza frequentemente fez uso da combinação de duas estratégias, a saber, o algoritmo mental (MA) e contagem nos dedos (COF) para resolver um mesmo cálculo, como em $34+55$. Além disso, utilizou uma estratégia do tipo compensação, onde primeiramente "chuta" um número e em seguida faz ajustes até obter a resposta correta. Esta estratégia foi utilizada para resolver uma subtração por meio de um cálculo de adição.

\section{Referências}

ALBERGARIA, I. S.; PONTE, J. P. Cálculo mental e calculadora. In CANAVARRO, A. P.; MOREIRA, D.; ROCHA, M. I. (Ed.). Tecnologias e educação matemática. Lisboa: SEM- 
SPCE, 2008, p. 98-109.

ANDRÉ, M. E. D. A. Estudo de caso em pesquisa e avaliação educacional. 3. ed. Brasília: Liber Livro Editora, 2008.

BARICCATTI, K. H. G. As relações entre as estratégias de resolução de cálculos mentais e escritos e os níveis de construção das operações aritméticas. 2010. Tese (Doutorado em educação) - Faculdade de Educação, Universidade Estadual de Campinas, Campinas.

BUENOS AIRES. Matemática: Cálculo mental con números naturales. Apuntes para la enseñanza. Gobierno de la Ciudad de Buenos Aires. Buenos Aires: Secretaria de Educación Dirección General de Planeamiento Dirección de Currícula, 2006.

BEISHUIZEN, M. Mental strategies and materials or models for addition and subtraction up to 100. In Dutch second grades. Journal for Research in Mathematics Education, 24(4), 1993, p. 294-323.

BEISHUIZEN, M. Different approaches to mastering mental calculation strategies. In: ANGHILERI, J. (Ed.), Principles and practices in arithmetic teaching - innovative approaches for the primary classroom, 2001, Buckingham: Open University Press, p. 119130.

BEISHUIZEN, M. The empty number line as a new model. In I. THOMPSON (Ed.), Issues in Teaching Numeracy in Primary schools, 2009, Buckingham: Open University Press, p. $157-168$.

BEISHUIZEN, M.; ANGHILERI, J. Which mental strategies in the early number curriculum? A comparison of British ideas and Dutch views. British Educational Research Journal, 24(5), 1998, p. 519-538.

BUYS, K. Mental arithmetic. In: VAN DEN HEUVEL-PANHUIZEN, M. (Ed.), Children learn mathematics: A learning-teaching trajectory with intermediate attainment targets for calculation with whole numbers in primary school. Netherlands: Sense Publishers, 2008, p. 121 - 146. (Obra original publicada em 2001).

FIORENTINI, D.; LORENZATO, S. Investigação em educação matemática: percursos teóricos e metodológicos. Campinas: Autores Associados, 2006.

GOMEZ CHACÓN, I. M. Matemática emocional: os afetos na aprendizagem matemática. Porto Alegre: Artmed, 2003.

LINS, R. C.; GIMENEZ, J. Perspectivas em aritmética e álgebra para o século XXI. Campinas: Papirus, 1997.

LUCANGELI, D.; TRESSOLDI, P. E.; BENDOTTI, M.; BONAMONI, M.; SIEGEL, L. S. Effective strategies for mental and written arithmetic calculation from the third to the fifth grade. Educational Psychology, vol. 23, n. 5, dez. de 2005, p. 507 - 520. 
MCINTOSH, A.; REYS, B. J.; REYS, R. E. A proposed framework for examining basic number sense. For the Learning of Mathematics, Canadá, v. 12, n. 3, p. 2-44, 1992.

MORAIS, C. M. S. O cálculo mental na resolução de problemas: um estudo no $1^{\mathrm{o}}$ ano de escolaridade. 2011. Dissertação (Mestrado em Educação Matemática) - Instituto Politécnico de Lisboa, Escola Superior de Educação de Lisboa, Lisboa.

PARRA, C. Cálculo mental na escola primária. In: PARRA, C.; SAIZ, I. (Org.). Didática da matemática: reflexões psicopedagógicas. Porto Alegre, RS: Artes Médicas, 1996. p. 186-235.

SERRAZINA, M. L. O sentido do número no $1^{\circ}$ ciclo: uma leitura de investigação. Boletim Gepem, Seropedica: Rio de Janeiro, n. 61, p.15-28, jul./dez. 2012.

SOWDER, J. Mental computation and number comparison: their roles in the development of number sense and computational estimation. In: HIEBERT, J.; BEHR, M. (Erds.). Number concepts and operations in the middle grades. Reston, VA: Lawrence Erlbaum, 1988, p. 182-197.

THOMPSON, I. Getting your head around mental calculation. In I. Thompson (Ed.), Issues in teaching numeracy in primary schools, 2009, Buckingham: Open University Press, p. $145-156$.

TREFFERS, A. Grade 1 (and 2) - Calculation up to 20. In: VAN DEN HEUVELPANHUIZEN, M. (Ed.), Children learn mathematics: A Learning-Teaching Trajectory with Intermediate Attainment Targets for Calculation with Whole Numbers in Primary School. Netherlands: Sense Publishers, 2008, p. 43 - 60. (Obra original publicada em 2001). 